\title{
Influence of Measurement Location on Local Crack Opening Displacement
}

\author{
ZhengMing Sun ${ }^{1}$ and Brigitte Weiss ${ }^{2}$ \\ ${ }^{1}$ National Institute of Advanced Industrial Science and Technology, Sendai 983-8551, Japan \\ ${ }^{2}$ Institute of Materials Physics, University of Vienna, Boltzmanngasse 5, A-1090 Wien, Austria
}

By means of local measurement with a laser interferometry technique, the difference in fatigue crack opening evaluation results was revealed when the measurements were conducted at different locations along the length of a fatigue crack in an aluminum alloy. It was found that the crack opening stress increases when the measuring location approaches the crack tip. The experimental study leads to a suggestion that the fatigue crack closure result evaluated with the data obtained from a location far from the crack tip will underestimate the closure effect.

(Received August 20, 2002; Accepted November 20, 2002)

Keywords: laser interference, crack opening displacement, fatigue, crack tip, aluminum

\section{Introduction}

Since the crack closure concept was first introduced by Elber, ${ }^{1)}$ the importance of crack closure phenomenon has been intensively studied ${ }^{2-7)}$ and many different methods or techniques have been established for the measurement of fatigue crack closure effect, including the crack opening displacement gauge method, the strain gauge method, ${ }^{2)}$ the ultrasonic method ${ }^{3)}$ the potential drop method ${ }^{4)}$ and acoustic emission technique. ${ }^{5)}$ In these methods, due to measuring locations or the certain width of the employed strain gauges, the information from the whole or part of the fatigue crack length was integrated and taken into consideration for the final evaluation to determine the crack opening point.

However, only the closure experienced in the vicinity of the crack tip may take effect on the growth behavior of a fatigue crack. Then we come to a question that how much this integrated signal coming from the crack length through measurement can represent the real situation at the crack tip. The purpose of the present study is to measure the crack opening data at different locations along the crack length with a laser interferometric technique ${ }^{8-10)}$ to further our understanding of the fatigue crack closure mechanisms and the current closure evaluation methods.

\section{Principle and Experiments}

The laser Interferometric Strain/Displacement Gauge (ISDG) is a laboratory tool that measures relative displacements or gauge lengths as short as $100 \mu \mathrm{m}$ with a least count resolution of approximately $2.5 \mathrm{~nm}$. The principle of ISDG is based on the diffraction and interference of the laser beams reflected from a pair of two parallel indentations made by a microhardness tester. These features make the ISDG useful in certain specialized studies of crack opening displacement and localized strain. A diffraction/interference pattern can be described as:

$$
I=I_{0} \sin ^{2} \beta / \beta^{2} \cos ^{2} \gamma
$$

where $\beta=\pi b \sin \theta / \lambda, \gamma=\pi d \sin \theta / \lambda, \lambda$ is the wavelength, $b$ is the dimension of the indentation, and $d$ is the distance between the two indentations. The incident beam is collimated with intensity $I_{0}$, and the intensity, $I$, of the interfered pattern varies with the angle $\theta$. The first part of eq. (1), $I_{0} \sin ^{2} \beta / \beta^{2}$, stands for the intensity variation of the diffracted beam, which has a first minimum at $\beta=+\pi$ and $-\pi$, and most energy of the radiation is included within this range. The employed fringe patterns for study will be in this range. With the second part of this equation, $\cos ^{2} \gamma$, involved, we have the interference pattern with a minimum whenever $\gamma=\pi / 2,3 \pi / 2,5 \pi / 2, \ldots$. Then the spacing between interference fringes is given by $\sin \theta=\lambda / d$. In short, the angular width of the principal maximum of the diffraction pattern is given by $\lambda / b$ and the angular spacing between interference fringes by $\lambda / d$. The position, $\theta$, of an interference fringe is then given by

$$
(m+1 / 2) \lambda=d \sin \theta ; \quad m=0,1,2,3 \ldots
$$

where $m$ is the order of the fringe and identifies each individual one. If $d$ changes a small amount, $\delta d$, the angular location of a given fringe moves by

$$
\delta \theta=-\delta d / d \tan \theta
$$

Note that the shape of the diffraction envelope has not changed because $b$ is kept constant. Thus a relative displacement between the two indentations causes the fringes to move within the diffraction envelope.

A 7475 aluminum alloy was used to study the crack closure behavior. Fatigue specimen with the gauge section of $6 \mathrm{~mm} \times 8 \mathrm{~mm}$ for four point bending tests was machined from a $6 \mathrm{~mm}$ thick plate. One of the surfaces was carefully polished before a semi-elliptical surface notch of $1.8 \mathrm{~mm}$ long and $0.9 \mathrm{~mm}$ deep was made in the centre of the width by spark erosion. Fatigue test was conducted at a stress ratio of -1 with a Schenck testing machine at a frequency of $25 \mathrm{~Hz}$. A load shedding procedure was employed to obtain a crack arrested at the fatigue threshold stress intensity.

Several pairs of microhardness indentations were introduced into the specimen surface across the crack. Figure 1 gives an example showing the locations (Fig. 1a) and the shape of the indentations (Fig. 1b). For the purpose to obtain a control example of the uncracked material another pair of indentations was also made on the extension line of the crack path, $0.5 \mathrm{~mm}$ ahead of the crack tip. Two strain gauges were pasted at two sides of the specimen with and without crack at the same corresponding position in order to check if the 

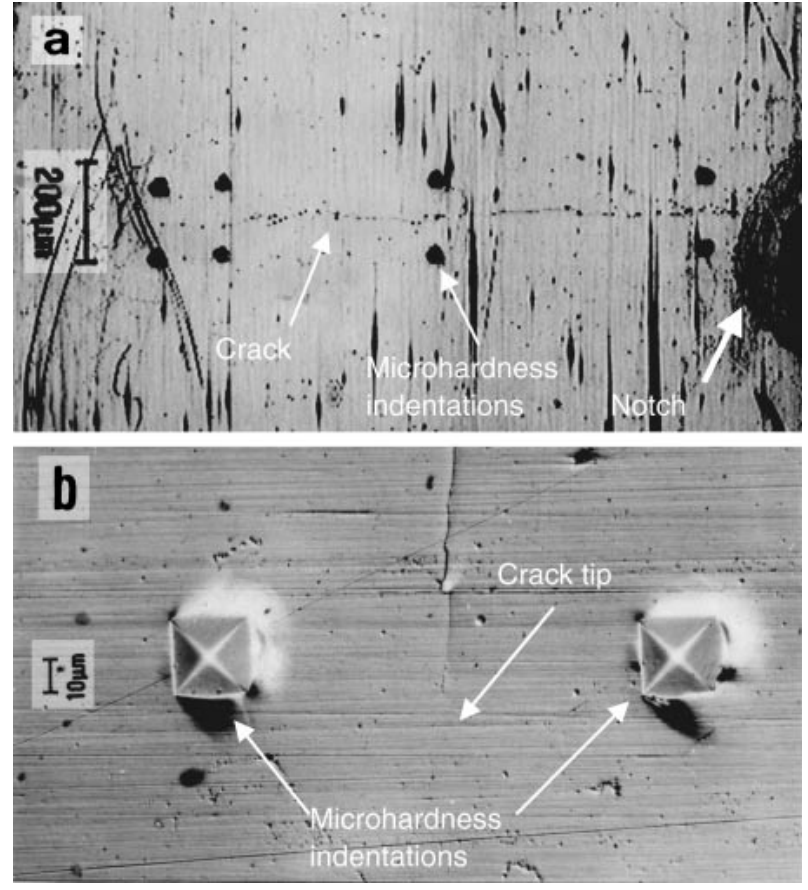

Fig. 1 An aluminum alloy specimen with a semi-elliptical surface fatigue crack showing, (a) the locations of the indentations and, (b) enlarged micrograph of a pair of indentations near the crack tip.

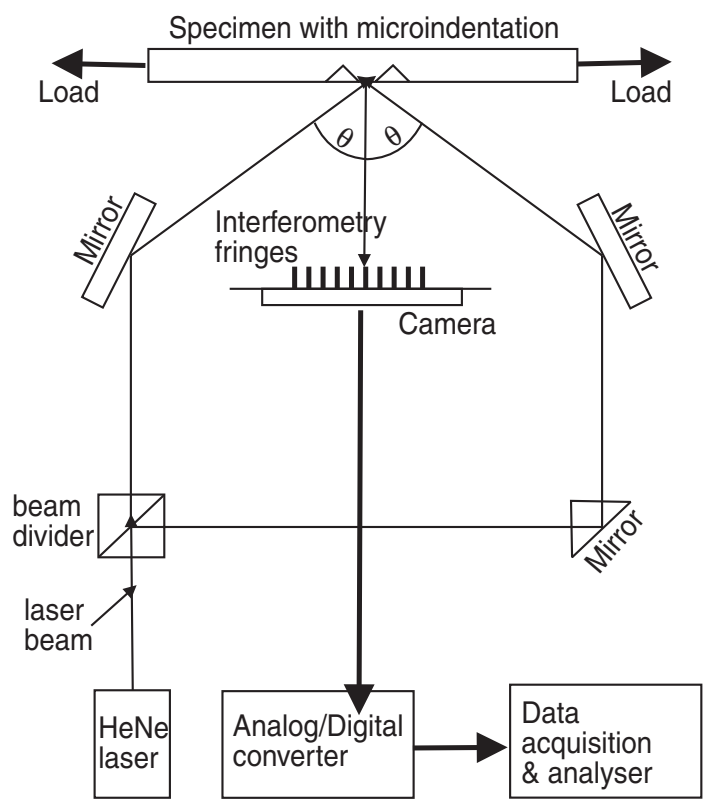

Fig. 2 Schematic setting of the laser interferometer for displacement measurement.

specimen is kept straight without misalignment. The set-up of the laser interferometry system used for this study is schematically illustrated in Fig. 2.

\section{Results and Discussion}

The results of the correlation between the applied load and the crack opening displacement (COD) determined with the ISDG from the specimen containing a semi-elliptical surface

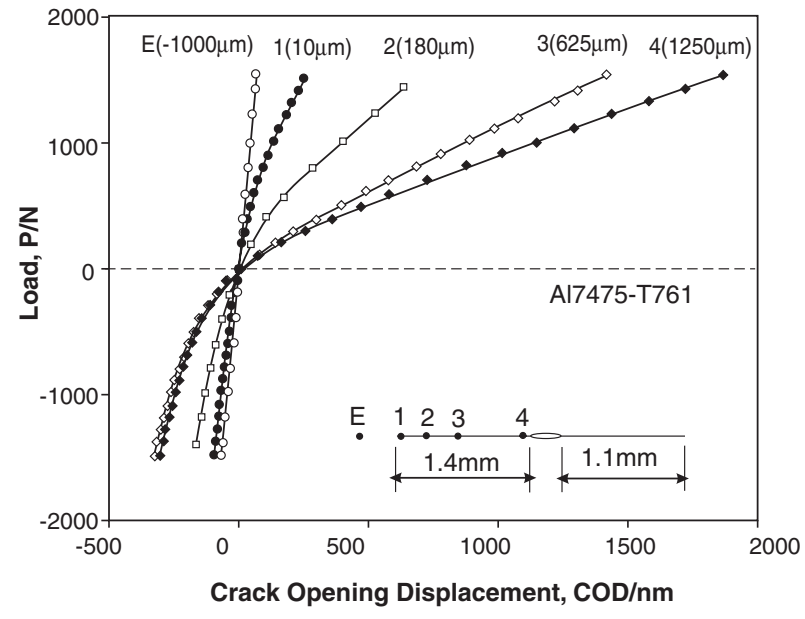

Fig. 3 Load-COD (crack opening displacement) curves for 7475-T761Al alloy at various measuring locations along the crack.

crack are shown in Fig. 3 where the locations of the measurements are indicated, with the fatigue crack tip being defined as zero point for the measuring location. From the load displacement measurements ahead of the crack tip, curve $E$, the value of the static Young's modulus for the solid material was deduced $(72 \mathrm{GPa})$. Curves $1-4$ represent the experimental data for the load-displacement measurements at $10 \mu \mathrm{m}, 180 \mu \mathrm{m}, 625 \mu \mathrm{m}$, and $1250 \mu \mathrm{m}$ behind the crack tip, respectively. The points indicate the relative change in distance between the pair of indentations with the distance at zero external load being defined as $\mathrm{COD}=0$. It is obvious that under tensile loading with increasing distance from the crack tip and with increasing load the crack opening increases. Furthermore, under compressive loading the crack was found to close at approximately $1000 \mathrm{~N}$. The behavior of a closed crack can be deduced from straight line relationship with increasing compressive load. It is interesting to note that such lines do not correspond exactly to the Young's modulus of the solid material (cure $E$ ) which may be explained by a certain amount of non-matching areas on the compressed fracture surfaces.

Crack opening load (or conventional crack closure load) is usually assumed to be that corresponding to the first contact of the fatigue crack surfaces in the unloading procedure. From the load displacement records, variations of the opening load, $P_{\mathrm{op}}$, were derived using the technique known as maximization of the correlation coefficient. ${ }^{11)}$ This technique involves taking the upper $10 \%$ of the loaddisplacement data and calculating the least squares correlation coefficient. The next data pair is then added and the correlation coefficient is again computed. This procedure is repeated for the whole data set. The point at which the correlation coefficient reaches a maximum can then be defined as $P_{\mathrm{op}}$, from which the opening stress $\sigma_{\mathrm{op}}$ can be derived. An evaluation of the present experimental results can be seen in Fig. 4 in which the determined opening stress, $\sigma_{\mathrm{op}}$, is not a constant but decreases with distance from the crack tip. Moreover, the values of $\sigma_{\text {op }}$ changes more sensitively when the measuring location approaches the crack tip. 


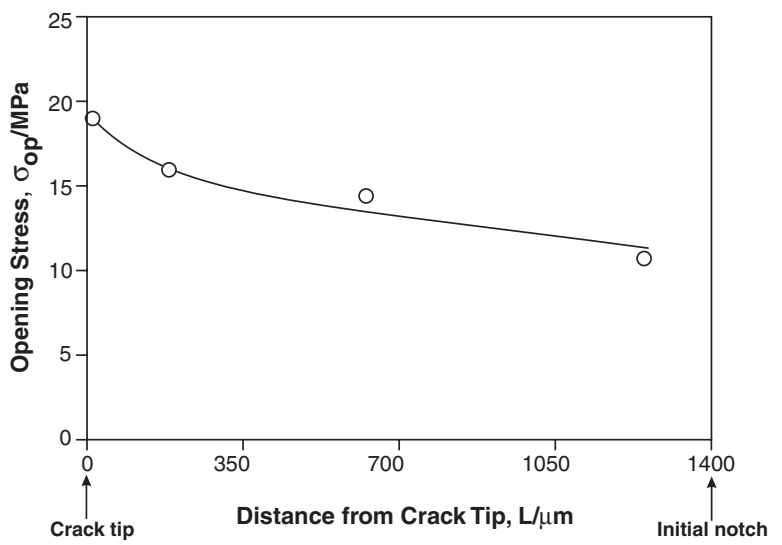

Fig. 4 Crack opening stress measured with laser interferometer as a function of the distance from the crack tip. law,

In regime II fatigue crack growth rate follows the Paris

$$
d a / d n=C(\Delta K)^{n}
$$

where $C$ and $n$ are considered to be material constants, $\Delta K$ is the stress intensity factor range, namely,

$$
\Delta K=K_{\max }-K_{\min } .
$$

During the process of fatigue loading fatigue crack surfaces may close at a stress intensity factor, $K_{\mathrm{op}}$, far higher than the $K_{\min }$. This leads to, with a first approximation or conventional definition, a reduction in the effective driving force for the fatigue crack growth,

$$
\Delta K=K_{\max }-K_{\mathrm{op}}
$$

The significance of $K_{\mathrm{op}}$ to fatigue crack growth is therefore obvious. However, how this $K_{\mathrm{op}}$ should be determined has long been an argument in the field of fatigue study. The experimental results of this study indicated that the determination of $K_{\mathrm{op}}$ is closely related to where the data are obtained, namely the location of measurement along the crack length. Local measurement of fatigue crack closure is therefore of great importance in further understanding the effective driving force of a fatigue crack and the intrinsic threshold values of a material.

A further observation of the fatigue crack closing procedure will help us to understand the results of the present investigation. Upon unloading of a specimen containing a fatigue crack the crack surfaces come into contact gradually. This point is already implied in the definition of the opening load. As the crack surfaces near the crack tip comes to contact at a higher load than the applied minimum load due to the causes such as crack tip plasticity or asperity in the crack wedge, the Load-COD curve measured close to the crack tip starts to deviate from linearity, while the crack surfaces far from the crack tip are still apart from each other (Fig. 3). When the measurements are conducted at these faraway locations the COD will decrease linearly with the load till a much lower lever as the contact at the tip will not influence the overall COD values which is of order lager in values. It is therefore reasonable to conclude that the data measured with the usual method are the averaged over these values, and hence lead to an underestimation of the crack closure effect.

\section{Summary}

Laser interferometry technique has been applied to the local measurement of fatigue crack closure effect. Experimental results of the measurement in a 7475 aluminum alloy indicated that the measurement location along the crack length has an effect on the result of fatigue crack opening points. The opening stress, $\sigma_{\mathrm{op}}$, is higher when the measurement was carried out near the crack tip while the value will decrease greatly with an increase in the distance from the crack tip. In other words, when the data obtained far from the crack tip or with a strain gauge method are used for closure assessment an effective stress intensity factor range larger than that actually experienced at the crack tip will be deduced. In other words, an underestimation of crack closure will be made when the crack opening displacement (COD) is not measured locally at the crack tip.

\section{REFERENCES}

1) E. Elber: Eng. Fract. Mech. 2 (1970) 37-45.

2) R. O. Ritchie, W. Yu and R. J. Bucci: Eng. Fract. Mech. 11 (1989) 361377.

3) C. L. Ho, O. Buck and H. L. Marcus: ASTM STP 536, (Philadelphia, PA, 1973) pp. 5-21.

4) V. Bachman and D. Munz: Fatigue Testing and Design 2 (1976) 351352.

5) C. S. Lee, C. G. Park and Y. W. Chang: Mater. Sci. Eng. A 216 (1996) 131-138.

6) C.-C. Chu and R. A. Chernenkoff: Int. J. Fatigue 23 (2001) 187-194.

7) S.-Y. Lee and J.-H. Song: Eng. Fract. Mech. 66 (2000) 321-346.

8) W. N. Sharpe Jr: Optical Eng. 21 (1982) 483-488.

9) B. Zagar, H. Weiss, D. L. Chen, H. Hoedl, B. Weiss and R. Stickler: Materwiss. Werkstofftech 23 (1992) 197-200.

10) A. H. Rosenberger and H. Ghonem: Fatigue Fract. Eng. Mater. Struct. 15 (1992) 1125-1139.

11) J. E. Allison, R. C. Ku and M. A. Pompetzki: ASTM STP982, (Philadelphia, PA, 1988) pp. 171-185. 